\title{
Smith-Magenis Syndrome
}

National Cancer Institute

\section{Source}

National Cancer Institute. Smith-Magenis Syndrome. NCI Thesaurus. Code C75469.

A genetic syndrome caused by an interstitial deletion in chromosome 17p11.2. It is characterized by mild to moderate mental retardation, distinctive facial features (flat head, square face, and deep set-eyes), sleep disturbances, attention deficit disorders, and temper tantrums. 